\title{
Combinatorial proofs of addition formulas
}

\author{
Xiang-Ke Chang Xing-Biao Hu \\ LSEC, ICMSEC, Academy of Mathematics and Systems Science \\ Chinese Academy of Sciences \\ Beijing, China \\ changxk, hxb@lsec.cc.ac.cn \\ Hongchuan Lei* \\ College of Science \\ Zhejiang Sci-Tech University \\ Hangzhou, China \\ hongchuanlei@gmail.com \\ Yeong-Nan Yeh \\ Institute of Mathematics \\ Academia Sinica \\ Taipei, Taiwan \\ mayeh@math.sinica.edu.tw
}

Submitted: Oct 29, 2014; Accepted: Jan 4, 2016; Published: Jan 11, 2016

Mathematics Subject Classifications: 05A19, 05A15, 15A15

\begin{abstract}
In this paper we give a combinatorial proof of an addition formula for weighted partial Motzkin paths. The addition formula allows us to determine the $L D U$ decomposition of a Hankel matrix of the polynomial sequence defined by weighted partial Motzkin paths. As a direct consequence, we get the determinant of the Hankel matrix of certain combinatorial sequences. In addition, we obtain an addition formula for weighted large Schröder paths.
\end{abstract}

Keywords: lattice paths; Catalan numbers; Motzkin numbers; Large Schröder numbers; Hankel determinant

\section{Introduction}

For a sequence $\left\{a_{n}\right\}_{n \geqslant 0}$, its $n$th Hankel matrix is defined to be the matrix $\left(a_{i, j}\right)_{0 \leqslant i, j \leqslant n-1}$, where $a_{i, j}=a_{i+j}$. There are many applications of Hankel matrices in combinatorics and in coding theory(see for instance $[17,22]$ ). The problem of evaluating the determinants of Hankel matrices (also called Hankel determinans for short) of a given sequence has been widely studied.

First, let's recall some classic results for sequences arising from enumerations of lattice paths. A Dyck path is a lattice path starting at $(0,0)$, ending at $(2 n, 0)$, and never going

\footnotetext{
*Corresponding author.
} 
below the $x$-axis, consisting of up steps $(1,1)$ and down steps $(1,-1)$. Let $c_{n}=\frac{1}{n+1}\left(\begin{array}{c}2 n \\ n\end{array}\right)$ be the Catalan number, it counts the number of Dyck paths from $(0,0)$ to $(2 n, 0)$. The Hankel determinants associated to the Catalan numbers are well studied (see $[9,15])$. It is well known that

$$
\begin{aligned}
& \operatorname{det}_{0 \leqslant i, j \leqslant n-1}\left(c_{i+j}\right)=1, \\
& \operatorname{det}_{0 \leqslant i, j \leqslant n-1}\left(c_{i+j+1}\right)=1, \\
& \operatorname{det}_{0 \leqslant i, j \leqslant n-1}\left(c_{i+j+m}\right)=\prod_{1 \leqslant i \leqslant j \leqslant m-1} \frac{2 n+i+j}{i+j} .
\end{aligned}
$$

A Motzkin path is a lattice path starting at $(0,0)$, ending at $(n, 0)$, and never going below the $x$-axis, consisting of up steps $(1,1)$, horizontal steps $(1,0)$ and down steps $(1,-1)$. Let $m_{n}=\sum_{k \geqslant 0}\left(\begin{array}{c}n \\ 2 k\end{array}\right) c_{k}$ be the Motzkin number, it counts the number of Motzkin paths from $(0,0)$ to $(n, 0)$. Let $M(z)=\sum_{n \geqslant 0} m_{n} z^{n}$ be the generating function, it is well known that

$$
M(z)=\frac{1-z-\sqrt{1-2 z-3 z^{2}}}{2 z^{2}} .
$$

The Hankel determinants associated to the Motzkin numbers are well studied by Aigner[1]

$$
\begin{aligned}
& \underset{0 \leqslant i, j \leqslant n-1}{\operatorname{det}}\left(m_{i+j}\right)=1, \\
& \underset{0 \leqslant i, j \leqslant n-1}{\operatorname{det}}\left(m_{i+j+1}\right)=\left\{\begin{array}{lll}
1 & \text { if } n \equiv 0,1 & (\bmod 6) \\
0 & \text { if } n \equiv 2,5 & (\bmod 6) \\
-1 & \text { if } n \equiv 3,4 & (\bmod 6) .
\end{array}\right.
\end{aligned}
$$

A large Schröder path is similar but allowing up steps $(1,1)$, double horizontal steps $(2,0)$ and down steps $(1,-1)$. The large Schröder numbers $r_{n}$ counts the number of large Schröder paths from $(0,0)$ to $(2 n, 0)$. It was proved in $[3,11]$ that

$$
\begin{aligned}
& \underset{0 \leqslant i, j \leqslant n-1}{\operatorname{det}}\left(r_{i+j}\right)=2^{\left(\begin{array}{c}
n \\
2
\end{array}\right)}, \\
& \operatorname{det}_{0 \leqslant i, j \leqslant n-1}\left(r_{i+j+1}\right)=2^{\left(\begin{array}{c}
n+1 \\
2
\end{array}\right) .}
\end{aligned}
$$

For Hankel determinants on small Schröder numbers please see [11]. Notice that by the Desnanot-Jacobi identity it is possible to determine $\operatorname{det}_{0 \leqslant i, j \leqslant n-1}\left(a_{i+j+m}\right)$ by $\operatorname{det}_{0 \leqslant i, j \leqslant n-1}\left(a_{i+j}\right)$ and $\operatorname{det}_{0 \leqslant i, j \leqslant n-1}\left(a_{i+j+1}\right)$. For more results on Hankel determinants of sequences related to the above sequences the reader may consult $[4,5,7,8,12,13,19,21]$.

In [18], C. Radoux presented several addition formulas for some polynomials building on combinatorial sequences. The formulas were proved by algebraic methods. In this paper, we mainly study the weighted partial Motzkin paths, also called weighted Motzkin path from $(0,0)$ to $(n, k)$ (see [6]). We generalize the addition formulas given by C. Radoux and provide a combinatorial proof. See Theorem 3 for our main result. 
The present paper is organized as follows. In Section 2, we define a unique decomposition of a lattice path into three parts. In Section 3, we first establish a bijection, and then prove the addition formula for the weighted partial Motzkin paths. In Section 4, by the addition formula, we obtain the $L D U$ decompositions of the Hankel matrices of the polynomial sequences, and evaluate the corresponding Hankel determinants. Then we specialize the parameters and get the Hankel determinants of several combinatorial sequences. Some of them are classic results but the others are new. In the last section, we deduce an addition formula for large Schröder paths, despite the lack of applications.

\section{Preliminaries}

In this article, let $\mathbb{Z}$ be the set of integers. For two points $P_{1}, P_{2} \in \mathbb{Z} \times \mathbb{Z}$, let $\overrightarrow{P_{1} P_{2}}$ denote the vector from $P_{1}$ to $P_{2}$.

Definition 1. Let $S$ be a finite set of vectors of $\mathbb{Z} \times \mathbb{Z}$. A lattice path $P$ with step set $S$, denoted by $\left(P_{0}, P_{1}, \ldots, P_{n}\right)$, is a path connecting the point sequence $P_{0}, P_{1}, \ldots, P_{n}$ such that each point $P_{i} \in \mathbb{Z} \times \mathbb{Z}$ and each step $\vec{P}_{i-1} P_{i} \in S$. The number $n$ is referred to as the length of $P$.

For a lattice path, the leftmost point and the rightmost point are called the start point and the end point respectively. The reverse path of a lattice path $P$, denoted by $\overleftarrow{P}$, is a lattice path obtained by reading $P$ from right to left with the same start point as $P$.

The $y$-coordinate of a point $P_{i}$ is called its height, denoted by $h\left(P_{i}\right)$. The concatenation of two lattice paths $P=\left(P_{0}, P_{1}, \ldots, P_{m}\right)$ and $P^{\prime}=\left(P_{0}^{\prime}, P_{1}^{\prime}, \ldots, P_{n}^{\prime}\right)$, denoted by $P \circ P^{\prime}$, is defined to be the path $\left(P_{0}, P_{1}, \ldots, P_{m}, P_{m}+\overrightarrow{P_{0}^{\prime} P_{1}^{\prime}}, P_{m}+\overrightarrow{P_{0}^{\prime} P_{2}^{\prime}}, \ldots, P_{m}+\overrightarrow{P_{0}^{\prime} P_{n}^{\prime}}\right)$. For two sets of lattice paths $\mathcal{P}$ and $\mathcal{P}^{\prime}$, let $\mathcal{P} \circ \mathcal{P}^{\prime}:=\left\{P \circ P^{\prime} \mid P \in \mathcal{P}, P^{\prime} \in \mathcal{P}^{\prime}\right\}$.

For a given step set $S$, let $\mathcal{P}_{n}^{k}$ denote the set of lattice paths from $(0,0)$ to $(n, k)$ constructed by steps in $S$ which never pass below the $x$-axis. Let

$$
\mathcal{P}_{n}^{\geqslant r}:=\bigcup_{k \geqslant r} \mathcal{P}_{n}^{k}
$$

Notice that the end point of each path in $\mathcal{P}_{n}^{\geqslant r}$ is on or above the line $y=r$.

For $P=\left(P_{0}, P_{1}, \ldots, P_{n}\right) \in \mathcal{P}_{n}^{k}$ and $0 \leqslant i \leqslant n$, let $L_{i}(P)$ denote the partial path of $P$ consisting of the first $i$ steps, i.e. $L_{i}(P)=\left(P_{0}, P_{1}, \ldots, P_{i}\right)$. For each $i \in\{0,1,2, \ldots, n\}$, there is a unique decomposition of $P$ :

$$
P=L_{i}(P) \circ M_{i}(P) \circ R_{i}(P) .
$$

where $M_{i}(P)=\left(P_{i}, P_{i+1}, \ldots, P_{j}\right), R_{i}(P)=\left(P_{j}, P_{j+1}, \ldots, P_{n}\right)$ and $P_{j}$ is the rightmost point of the partial path $\left(P_{i}, P_{i+1}, \ldots, P_{n}\right)$ with the smallest height. See Figure 1 for an illustration. 


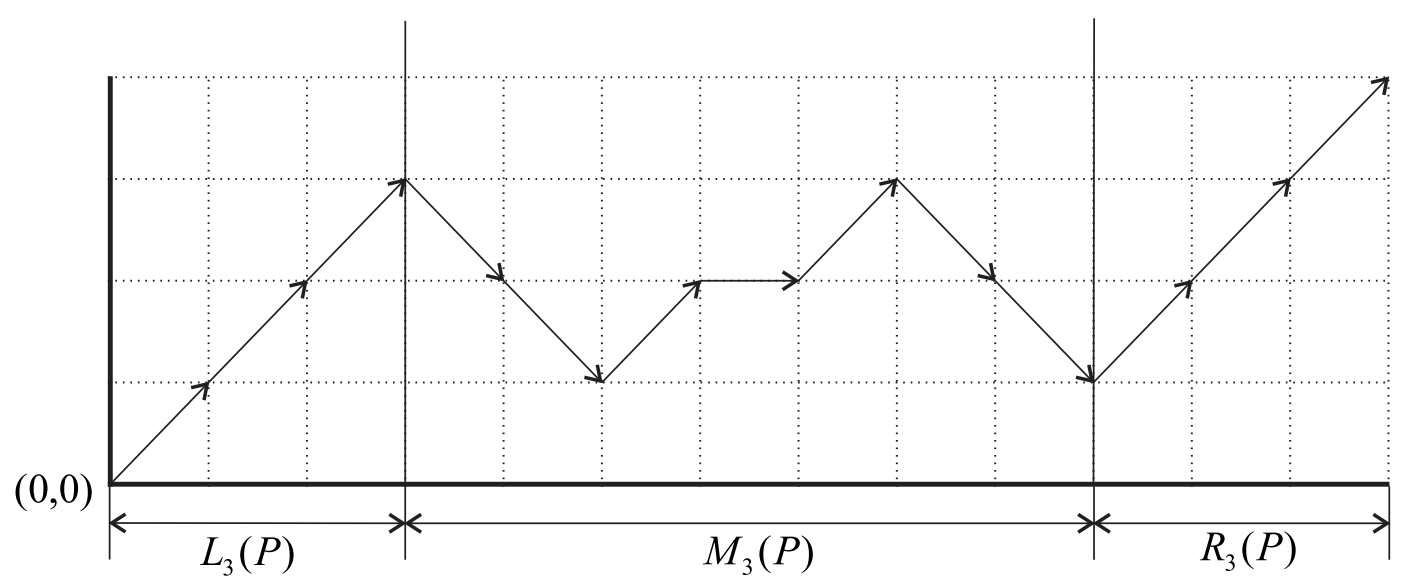

Figure 1: For $i=3, P=L_{3}(P) \circ M_{3}(P) \circ R_{3}(P)$

\section{An addition formula for weighted partial Motzkin paths}

In this section, we study weighted partial Motzkin paths. Let the step set $S=\{(1,1)$, $(1,-1),(1,0)\}$. A path in $\mathcal{P}_{n}^{k}$ is called a weighted partial Motzkin path if each step has been assigned a weight. In the following, we let

$$
w(1,1)=x y, w(1,0)=u x, w(1,-1)=v x / y .
$$

For a path $P \in \mathcal{P}_{n}^{k}$, the weight should be $u^{r} v^{s} x^{n} y^{k}$, where $x$ and $y$ mark the coordinates of the end point, $u$ marks the horizontal steps $(1,0)$, and $v$ marks the down steps $(1,-1)$. We immediately have $w\left(P \circ P^{\prime}\right)=w(P) w\left(P^{\prime}\right)$ for any two lattice paths $P$ and $P^{\prime}$. The weight of a lattice path set is the sum of the weights of all its paths.

Let

$$
a_{n, k}:=\frac{1}{x^{n} y^{k}} w\left(\mathcal{P}_{n}^{k}\right)
$$

For the case $u=v=1, a_{n, 0}$ is the $n$th Motzkin number and the matrix $\left(a_{i, j}\right)_{0 \leqslant i, j \leqslant n-1}$ is the Motzkin triangle (the sequence A026300 in [20]). For the case $u=0$ and $v=1$, $a_{2 n, 0}$ is the $n$th Catalan number and $\left(a_{i, j}\right)_{0 \leqslant i, j \leqslant n-1}$ is the Catalan triangle (the sequence A053121 in [20]).

For the path set $\mathcal{P}_{n}^{\geqslant r}$, define its generating function as

$$
a_{n, r}(y):=\frac{1}{x^{n} y^{r}} w\left(\mathcal{P}_{n}^{\geqslant r}\right)
$$

It is obvious that $a_{n, r}(y)=\sum_{k \geqslant r} a_{n, k} y^{k-r}$. Here $y$ marks the distance from the end point of each path in $\mathcal{P}_{n}^{\geqslant r}$ to the line $y=r$.

For fixed nonnegative integers $m$ and $n$, we denote by $\mathcal{Q}_{r}$ the set of all the paths $P=\left(P_{0}, P_{1}, \ldots, P_{m+n}\right)$ in $\mathcal{P}_{m+n}^{\geqslant 0}$ satisfying that

$$
h\left(P_{m}\right)-h\left(P_{j}\right)=r,
$$


where $j$ is determined by setting $i=m$ and replacing $n$ by $m+n$ in (1). It's easy to see that $\left\{\mathcal{Q}_{0}, \mathcal{Q}_{1}, \ldots, \mathcal{Q}_{\min (m, n)}\right\}$ is a partition of $\mathcal{P}_{m+n}^{\geqslant 0}$.

The next lemma provides the key bijection of our main theorem.

Lemma 2. For $0 \leqslant r \leqslant \min (m, n)$, define $\phi_{r}: \mathcal{Q}_{r} \rightarrow \mathcal{P}_{m}^{\geqslant r} \circ \mathcal{P}_{n}^{\geqslant r}$ by

$$
P \rightarrow \phi_{r}(P)=L_{m}(P) \circ \overleftarrow{M_{m}(P)} \circ R_{m}(P)
$$

Then $\phi_{r}$ is a bijection and $w(P)=\frac{v^{r}}{y^{2 r}} w\left(\phi_{r}(P)\right)$.

Proof. Since the decomposition (1) is unique for $i=m$, it is obvious that $\phi_{r}$ is an injection. We only need to find $\phi_{r}^{-1}$. Given two paths $P \in \mathcal{P}_{m}^{\geqslant r}$ and $P^{\prime} \in \mathcal{P}_{n}^{\geqslant r}$, we first split $P^{\prime}$ into two subpaths $P_{1}^{\prime}$ and $P_{2}^{\prime}$ at its rightmost point with height $r$ such that $P^{\prime}=P_{1}^{\prime} \circ P_{2}^{\prime}$. Then $\phi_{r}^{-1}\left(P \circ P^{\prime}\right)=P \circ \overleftarrow{P_{1}^{\prime}} \circ P_{2}^{\prime}$. See Figure 2 for an example.

Observe that $P$ has $r$ more down steps than $\phi_{r}(P)$, and the same number of horizontal steps, so we have $w(P)=\frac{v^{r}}{y^{2 r}} w\left(\phi_{r}(P)\right)$.

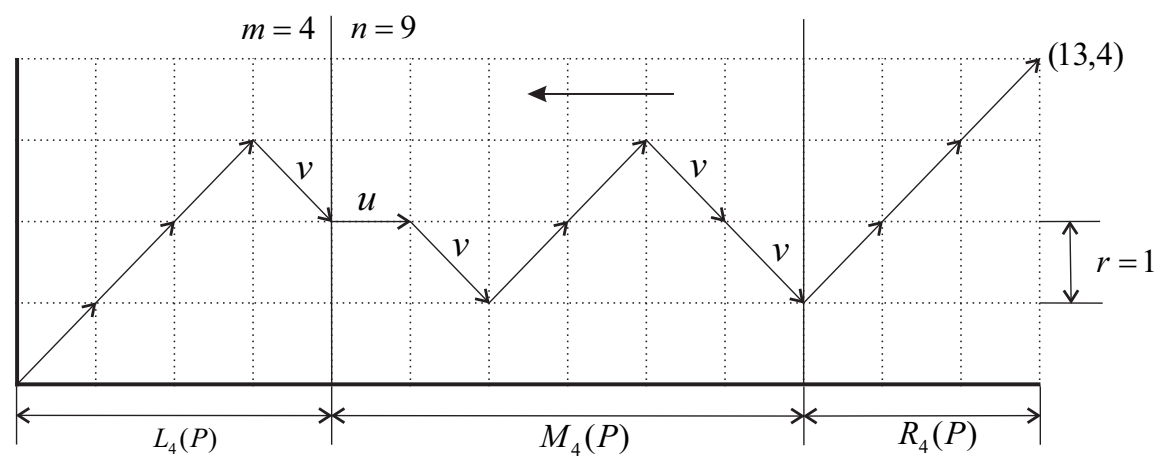

1)

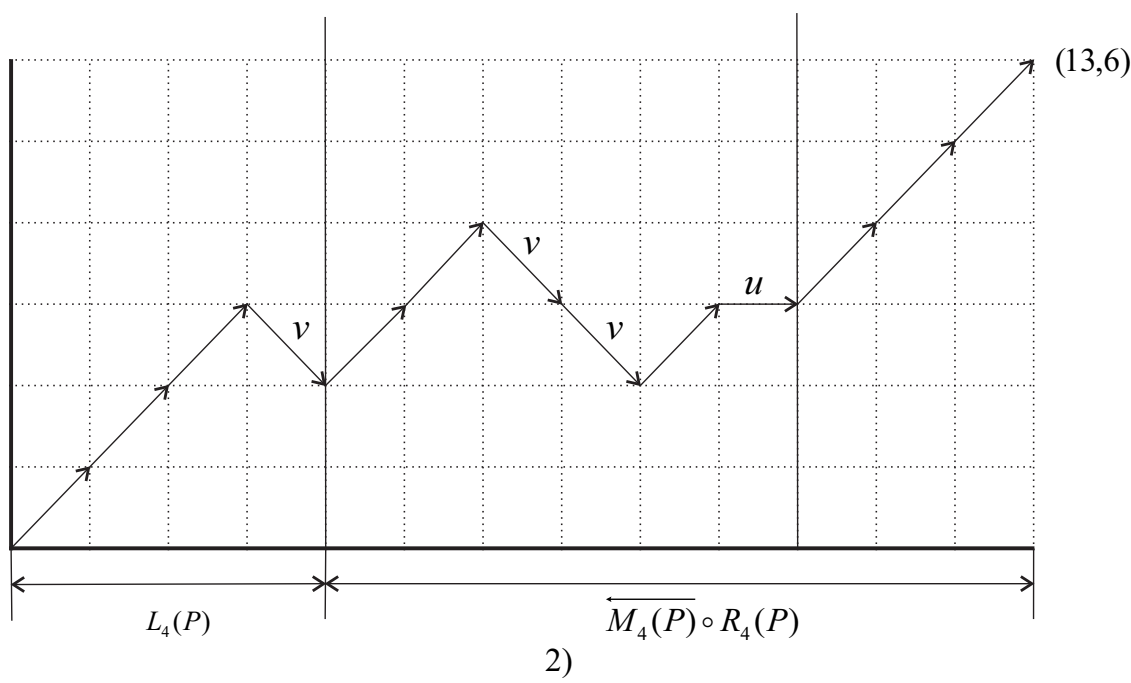

Figure 2: 1). $\left.P \in \mathcal{Q}_{1} ; 2\right) . \phi(P) \in \mathcal{P}_{4}^{\geqslant 1} \circ \mathcal{P}_{9}^{\geqslant 1}$ 
Now we prove the main theorem of this paper.

Theorem 3. For nonnegative integers $m$ and $n$, we have

$$
a_{m+n, 0}(y)=\sum_{r=0}^{\min (m, n)} v^{r} a_{m, r}(y) a_{n, r}(y) .
$$

Proof. By Lemma 2, we have

$$
w\left(\mathcal{Q}_{r}\right)=\frac{v^{r}}{y^{2 r}} w\left(\mathcal{P}_{m}^{\geqslant r}\right) w\left(\mathcal{P}_{n}^{\geqslant r}\right)
$$

Therefore

$$
\begin{aligned}
a_{m+n, 0}(y) & =\frac{1}{x^{m+n}} w\left(\mathcal{P}_{m+n}^{\geqslant 0}\right)=\frac{1}{x^{m+n}} \sum_{r=0}^{\min (m, n)} w\left(\mathcal{Q}_{r}\right) \\
& =\sum_{r=0}^{\min (m, n)} v^{r}\left(\frac{1}{x^{m} y^{r}} w\left(\mathcal{P}_{m}^{\geqslant r}\right)\right)\left(\frac{1}{x^{n} y^{r}} w\left(\mathcal{P}_{n}^{\geqslant r}\right)\right) \\
& =\sum_{r=0}^{\min (m, n)} v^{r} a_{m, r}(y) a_{n, r}(y) .
\end{aligned}
$$

Example 4. Let $u=v=1$. Then $a_{i, j}$ is the number of lattice paths with step set $S=\{(1,1),(1,0),(1,-1)\}$ begin at the origin and end at $(i, j)$ that never pass below the $x$-axis. By direct calculation we have

$$
\begin{aligned}
\left(a_{i, j}\right)_{0 \leqslant i, j \leqslant 4} & =\left(\begin{array}{lllll}
1 & 0 & 0 & 0 & 0 \\
1 & 1 & 0 & 0 & 0 \\
2 & 2 & 1 & 0 & 0 \\
4 & 5 & 3 & 1 & 0 \\
9 & 12 & 9 & 4 & 1
\end{array}\right), \\
\left(a_{i, j}(y)\right)_{0 \leqslant i, j \leqslant 4} & = \\
& \left(\begin{array}{llllll}
1 & & & & \\
y+1 & 1 & 0 & & \\
y^{2}+2 y+2 & y+2 & 0 & 0 & 0 \\
y^{3}+3 y^{2}+5 y+4 & y^{2}+3 y+5 & 1 & 0 & 0 \\
y^{4}+4 y^{3}+9 y^{2}+12 y+9 & y^{3}+4 y^{2}+9 y+12 & y^{2}+4 y+9 & y+4 & 1
\end{array}\right)
\end{aligned}
$$

and $a_{5,0}(y)=y^{5}+5 y^{4}+14 y^{3}+25 y^{2}+30 y+21$. It is direct to check that

$$
a_{5,0}(y)=\sum_{r=0}^{\min (m, n)} a_{m, r}(y) a_{n, r}(y)
$$

is true for each $(m, n) \in\{(0,5),(1,4),(2,3),(3,2),(4,1),(5,0)\}$. 
A direct consequence of Theorem 3 is the following result involving Catalan numbers. It was proved in [18] by algebraic method.

Corollary 5. [18] Let $c_{n, k}$ be the number of lattice paths from $(0,0)$ to $(2 n, 2 k)$ using steps $(1,1)$ and $(1,-1)$ that never pass below the $x$-axis. In particular, $c_{n, 0}$ is the $n$th Catalan number. Let

$$
c_{n, r}(y):=\sum_{i=r}^{n} c_{n, i} y^{i-r} \text {. }
$$

Then

$$
c_{m+n, 0}(y)=c_{m, 0}(y) c_{n, 0}(y)+(y+1) \sum_{r=1}^{\min (m, n)} c_{m, r}(y) c_{n, r}(y),
$$

for nonnegative integers $m$ and $n$.

Proof. Let $u=0$ and $v=1$ in (2), then $a_{2 n, 2 i}=c_{n, i}$. Since $a_{2 n, j}=0$ for odd $j$, then $a_{2 n, 2 r}(y)=c_{n, r}\left(y^{2}\right)$ and $a_{2 n, 2 r-1}(y)=y a_{2 n, 2 r}(y)$ for $r \geqslant 1$. By Theorem 3, we have

$$
\begin{aligned}
c_{m+n, 0}\left(y^{2}\right) & =a_{2 m+2 n, 0}(y)=\sum_{r=0}^{\min (2 m, 2 n)} a_{2 m, r}(y) a_{2 n, r}(y) \\
& =a_{2 m, 0}(y) a_{2 n, 0}(y)+\sum_{r=1}^{\min (m, n)}\left(a_{2 m, 2 r-1}(y) a_{2 n, 2 r-1}(y)+a_{2 m, 2 r}(y) a_{2 n, 2 r}(y)\right) \\
& =c_{m, 0}\left(y^{2}\right) c_{n, 0}\left(y^{2}\right)+\left(y^{2}+1\right) \sum_{r=1}^{\min (m, n)} c_{m, r}\left(y^{2}\right) c_{n, r}\left(y^{2}\right) .
\end{aligned}
$$

Replacing $y^{2}$ by $y$ completes the proof.

\section{Applications}

In this section, we show that equation (3) can be used to evaluate many Hankel determinants of certain sequences.

Theorem 6. Let $A_{n}(y)$ denote the $n$th Hankel matrix of the sequence $\left\{a_{n, 0}(y)\right\}_{n \geqslant 0}$, i.e.

$$
A_{n}(y)=\left(a_{i+j, 0}(y)\right)_{0 \leqslant i, j \leqslant n-1} .
$$

Then

$$
\operatorname{det}\left(A_{n}(y)\right)=v^{\frac{n(n-1)}{2}}
$$

Proof. By Theorem 3, we have

$$
A_{n}(y)=A \cdot \operatorname{diag}\left(v^{0}, v^{1}, \ldots, v^{n-1}\right) \cdot A^{T}
$$


where $A$ is the following lower triangular matrix:

$$
A=\left(\begin{array}{ccccc}
a_{0,0}(y) & & & & \\
a_{1,0}(y) & a_{1,1}(y) & & & \\
a_{2,0}(y) & a_{2,1}(y) & a_{2,2}(y) & & \\
\cdots & \cdots & \cdots & \cdots & \\
a_{n-1,0}(y) & a_{n-1,1}(y) & a_{n-1,2}(y) & \cdots & a_{n-1, n-1}(y)
\end{array}\right) .
$$

So that

$$
\operatorname{det}\left(A_{n}(y)\right)=\operatorname{det}\left(\operatorname{diag}\left(v^{0}, v^{1}, \ldots, v^{n-1}\right)\right)(\operatorname{det}(A))^{2}=v^{\frac{n(n-1)}{2}}\left(\prod_{i=0}^{n-1} a_{i, i}(y)\right)^{2},
$$

and the proof follows immediately from the fact that $a_{i, i}(y)=a_{i, i}=1$ for $0 \leqslant i \leqslant n-1$.

For the weighted partial Motzkin paths, the above result shows that the determinant of $A_{n}(y)$ is independent of $u$ and $y$. Now in the following corollaries we specialize $y, u$ and $v$ in Theorem 6 , and the we obtain the Hankel determinants of several combinatorial sequences. Some results are known, only partial of them are listed. We also get some new results(see Corollary 8(2), (3) and Corollary 9(4)). The proofs are not difficult, so we leave them to the reader.

Corollary 7. Let $y=0$ in Theorem 6 , then $a_{n, 0}(0)=a_{n, 0}$ and $x^{n} a_{n, 0}$ is the weight of Motzkin paths of length $n$. Furthermore,

(1) [2] let $u=0$ and $v=1$, then $a_{2 n, 0}$ is the $n$th Catalan number, $a_{2 n-1}=0$ for $n \geqslant 1$, and

$$
\operatorname{det}_{0 \leqslant i, j \leqslant n-1}\left(a_{i+j, 0}\right)=1
$$

(2) [1] let $u=1$ and $v=1$, then $a_{n, 0}$ is the nth Motzkin number, and

$$
\operatorname{det}_{0 \leqslant i, j \leqslant n-1}\left(a_{i+j, 0}\right)=1 \text {; }
$$

(3) [10] let $u=1$ and $v=2$, then $a_{n, 0}$ is the number of increasing unary-binary trees with associated permutation that avoids 231 (the sequence A025235 in [20]), and

$$
\operatorname{det}_{0 \leqslant i, j \leqslant n-1}\left(a_{i+j, 0}\right)=2^{\frac{n(n-1)}{2}} ;
$$

(4) [14] let $u=1$ and $v=3$, then $a_{n, 0}$ is the number of lattice paths within $N^{2}$ starting at the origin and ending on the $y$-axis with step set $\{(-1,-1),(-1,0),(-1,1),(0,1)$, $(1,1)\}$. Also, $a_{n, 0}$ is the number of lattice paths within $N^{3}$ starting at the origin and consisting of $n$ steps taken from $\{(-1,-1,1),(-1,0,1),(-1,1,1),(0,0,-1),(1,1,0)\}$ (the sequence A025237 in [20]), and

$$
\operatorname{det}_{0 \leqslant i, j \leqslant n-1}\left(a_{i+j, 0}\right)=3^{\frac{n(n-1)}{2}} .
$$


Corollary 8. Let $y=1$ in Theorem 6 , then $a_{n, 0}(1)=\sum_{k=0}^{n} a_{n, k}$. Furthermore,

(1) [16] let $u=1$ and $v=1$, then $a_{n, 0}(1)$ is the number of permutations on $[n+1]$ that avoid the patterns 1-23-4 and 1-3-2, where the omission of a dash in a pattern means the permutation entries must be adjacent (the sequence A005773 in [20]), and

$$
\operatorname{det}_{0 \leqslant i, j \leqslant n-1}\left(a_{i+j, 0}(1)\right)=1 \text {; }
$$

(2) let $u=1$ and $v=3$, then $a_{n, 0}(1)$ is the number of lattice paths within $N^{2}$ starting at the origin and consisting of $n$ steps taken from $\{(-1,-1),(-1,1),(-1,0),(0,1),(1,1)\}$ (the sequence A151292 in [20]), and

$$
\operatorname{det}_{0 \leqslant i, j \leqslant n-1}\left(a_{i+j, 0}(1)\right)=3^{\frac{n(n-1)}{2}} ;
$$

(3) let $u=2$ and $v=2$, then $a_{n, 0}(1)$ is the number of lattice paths within $N^{3}$ starting at the origin and consisting of $n$ steps taken from $\{(-1,-1,-1),(-1,-1,0),(0,0,1)$, $(0,1,0),(1,1,1)\}$ (the sequence A151090 in [20]), and

$$
\operatorname{det}_{0 \leqslant i, j \leqslant n-1}\left(a_{i+j, 0}(1)\right)=2^{\frac{n(n-1)}{2}} .
$$

The most interesting case is $y=-1$. If $u=2$ and $v=1$, then $a_{n, 0}(-1)$ is the $n$th Catalan number. If $u=3$ and $v=2$, then $a_{n, 0}(-1)$ is the $n$th large Schröder number. We list some of the other cases below.

Corollary 9. Let $y=-1$ in Theorem 6, then $a_{n, 0}(-1)=\sum_{k=0}^{n}(-1)^{k} a_{n, k}$. Furthermore,

(1) [16] let $u=1$ and $v=1$, then $a_{n, 0}(-1)$ is the number of ordered trees with $n$ edges and no vertices of outdegree 1 . Also, $a_{n, 0}(-1)$ is number of 321-avoiding permutations on $[n]$ in which each left-to-right maximum is a descent (the sequence A005043 in [20]), and

$$
\operatorname{det}_{0 \leqslant i, j \leqslant n-1}\left(a_{i+j, 0}(-1)\right)=1 \text {; }
$$

(2) [16] let $u=3$ and $v=1$, then $a_{n, 0}(-1)$ is the number of binary trees of weight $n$ where leaves have positive integer weights. Also, $a_{n, 0}(-1)$ is the number of 321avoiding partitions of $[n]$ (the sequence A007317 in [20]), and

$$
\operatorname{det}_{0 \leqslant i, j \leqslant n-1}\left(a_{i+j, 0}(-1)\right)=1 \text {; }
$$

(3) (by Paul Barry, see A052709 in [20]) let $u=2$ and $v=2$, then $a_{n, 0}(-1)$ is the number of lattice paths starting from the origin to $(n, 0)$ with step set $\{(1,1),(1,-1)$, $(0,-1)\}$, and

$$
\operatorname{det}_{0 \leqslant i, j \leqslant n-1}\left(a_{i+j, 0}(-1)\right)=2^{\frac{n(n-1)}{2}} ;
$$

(4) let $u=4$ and $v=4$, then $a_{n, 0}(-1)$ is the number of Dyck paths with $2 n+2$ steps in which each up step not at the $x$-axis has two colors (the sequence A064062 in [20]), and

$$
\operatorname{det}_{0 \leqslant i, j \leqslant n-1}\left(a_{i+j, 0}(-1)\right)=4^{\frac{n(n-1)}{2}} .
$$




\section{Another addition formula}

In this section, we give an addition formula for weighted large Schröder paths, despite the lack of applications. Let the step set $S=\{(1,1),(1,-1),(2,0)\}$. The weights of the steps are given by

$$
w(1,1)=x y, w(2,0)=u x^{2}, w(1,-1)=v x / y .
$$

In this case, we denote

$$
r_{n, k}:=\frac{1}{x^{n} y^{k}} w\left(\mathcal{P}_{n}^{k}\right), r_{n, r}(y):=\frac{1}{x^{n} y^{r}} w\left(\mathcal{P}_{n}^{\geqslant r}\right) .
$$

Then $r_{n, s}(y)=\sum_{k=s}^{n} r_{n, k} y^{k-s}$. For the case $u=v=1, r_{2 n, 0}$ is the $n$th large Schröder number (the sequence A006318 in [20]).

Next we have the addition formula for large Schröder numbers.

Theorem 10. For $m, n \geqslant 1$,

$$
r_{m+n, 0}(y)=u \sum_{s=0}^{\min (m-1, n-1)} v^{s} r_{m-1, s}(y) r_{n-1, s}(y)+\sum_{s=0}^{\min (m, n)} v^{s} r_{m, s}(y) r_{n, s}(y) .
$$

Proof. The proof is very similar to that of Theorem 3. We divide $\mathcal{P}_{m+n}^{\geqslant 0}$ into two subsets: the first one is the set of paths crossing the line $x=m$ by a $(2,0)$-step, and the other one contains paths touching the line $x=m$. Then the first term of the right hand side of (4) represents the first subset and the second term represents the other subset. See Figure 3 for an illustration for the bijection of the first subset. The second one is the same as in Lemma 2.

Example 11. Let $u=v=1, r_{n, k}$ is the number of lattice paths with step set $S=\{(1,1)$, $(2,0),(1,-1)\}$ begin at the origin and end at $(n, k)$ that never pass below the $x$-axis. By direct calculation we have

$$
\begin{aligned}
& \left(r_{i, j}\right)_{0 \leqslant i, j \leqslant 5}=\left(\begin{array}{llllll}
1 & 0 & 0 & 0 & 0 & 0 \\
0 & 1 & 0 & 0 & 0 & 0 \\
2 & 0 & 1 & 0 & 0 & 0 \\
0 & 4 & 0 & 1 & 0 & 0 \\
6 & 0 & 6 & 0 & 1 & 0 \\
0 & 16 & 0 & 8 & 0 & 1
\end{array}\right) \\
& \left(r_{i, j}(y)\right)_{0 \leqslant i, j \leqslant 5}= \\
& \left(\begin{array}{llllll}
1 & 0 & 0 & 0 & 0 & 0 \\
y & 1 & 0 & 0 & 0 & 0 \\
y^{2}+2 & y & 1 & 0 & 0 & 0 \\
y^{3}+4 y & y^{2}+4 & y & 1 & 0 & 0 \\
y^{4}+6 y^{2}+6 & y^{3}+6 y & y^{2}+6 & y & 1 & 0 \\
y^{5}+8 y^{3}+16 y & y^{4}+8 y^{2}+16 & y^{3}+8 y & y^{2}+8 & y & 1
\end{array}\right)
\end{aligned}
$$



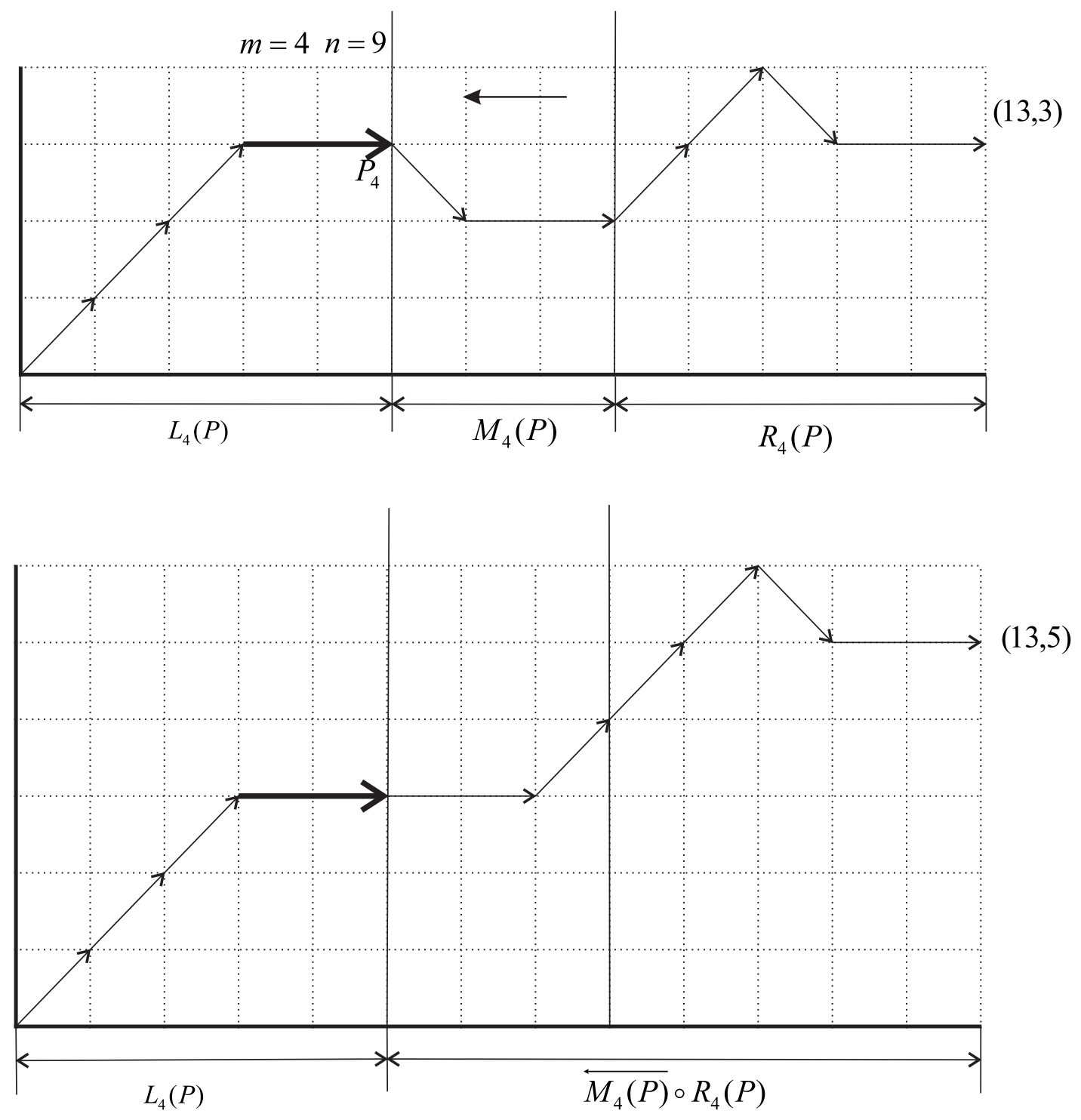

Figure 3: A lattice path $P \in \mathcal{P}_{13}^{3}$ 
and $r_{6,0}(y)=y^{6}+10 y^{4}+30 y^{2}+22$. It is easy to check that the identity

$$
r_{6,0}(y)=\sum_{s=0}^{\min (m-1, n-1)} r_{m-1, s}(y) r_{n-1, s}(y)+\sum_{s=0}^{\min (m, n)} v^{s} r_{m, s}(y) r_{n, s}(y)
$$

holds for $(m, n) \in\{(1,5),(2,4),(3,3),(4,2),(5,1)\}$.

\section{Acknowledgements}

The authors would like to express their deep gratefulness to the reviewers for their detail comments and valuable suggestions. Xing-Biao Hu was supported by Natural Science Foundation of Shandong Province of China ZR2014AM001. Yeong-Nan Yeh was supported by NSC 104-2115-M-001-010-MY3.

\section{References}

[1] M. Aigner. Motzkin numbers. European J. of Comb., 19:663-675, 1998.

[2] R. Bojičić, M. D. Petković, and P. Barry. The Hankel transform of aerated sequences. Integral Transforms and Special Functions, 24(9):685-699, 2013.

[3] R. A. Brualdi and S. Kirkland. Aztec diamonds and digraphs, and Hankel determinants of Schröder numbers. J. Combin. Theory Ser. B, 94:334-351, 2005.

[4] N. T. Cameron and A. C. M. Yip. Hankel determinants of sums of consecutive Motzkin numbers. Linear Algebra Appl., 434:712-722, 2011.

[5] X. K. Chang, X. B. Hu and Y. N. Zhang. A direct method for evaluating some nice Hankel determinants and proofs of several conjectures. Linear Algebra Appl., 4389:2523-2541, 2013.

[6] William Y.C. Chen, Nelson Y. Li, Louis W. Shapiro, and Sherry H.F. Yan. Matrix identities on weighted partial Motzkin paths. European J. Combin., 28(4):1196-1207, 2007.

[7] J. Cigler and C. Krattenthaler. Some determinants of path generating functions. Adv. Appl. Math., 46:144-174, 2011.

[8] A. Cvetković, P. Rajković, and M. Ivković. Catalan numbers, the Hankel transform, and Fibonacci numbers. J. Integer Seq., 5:Article 02.1.3, 2002.

[9] M. Desainte-Catherine and G. Viennot. Enumeration of certain Young tableaux with bounded height, Combinatoire Énumérative. Lecture Notes in Mathematics, Springer, Berlin, 1234:58-67, 1986.

[10] M. Dziemiańczuk. Counting Lattice Paths With Four Types of Steps. Graphs Comb., 30(5):1427-1452, 2013.

[11] S. P. Eu and T. S. Fu. A simple proof of the Aztec diamond theorem. Electron. J. Combin., 12:R18, 2005. 
[12] S. P. Eu, T. L. Wong and P. L. Yen. Hankel determinants of sums of consecutive weighted Schröder numbers. Linear Algebra Appl., 437:2285-2299, 2012.

[13] I. Gessel and X. Viennot. Binomial determinants, paths, and hook length formulae. Adv. Math., 58:300-321, 1985.

[14] A. Hennessy. A study of Riordan arrays with applications to continued fractions, orthogonal polynomials and lattice paths. PhD Thesis, Waterford Institute of Technology, 2011.

[15] C. Krattenthaler. Advanced determinant calculus: A complement. Linear Algebra Appl., 411:68-166, 2005.

[16] J. W. Layman. The Hankel transform and some of its properties. J. Integer Seq., $4: 1-11,2001$.

[17] H. Liang, L. Mu and Y. Wang. Catalan-like numbers and Stieltjes moment sequences. Discrete Math., 339(2):484-488, 2016.

[18] C. Radoux. Addition formulas for polynomials built on classical combinatorial sequences. J. Comput. Appl. Math., 115:471-477, 2000.

[19] P. M. Rajković, M. D. Petković and P. Barry. The Hankel transform of the sum of consecutive generalized Catalan numbers. Integral Transform. Spec. Funct., 18:285296, 2007.

[20] N. J. A. Sloane. On-line Encyclopedia of Integer Sequences (OEIS). Published electronically at http://oeis.org/, 2014.

[21] R. A. Sulanke and G. Xin. Hankel determinants for some common lattice paths. Adv. Appl. Math., 40:149-167, 2008.

[22] U. Tamm. Some Aspects of Hankel Matrices in Coding Theory and Combinatorics. Electron. J. Combin., 8:A1, 2001. 